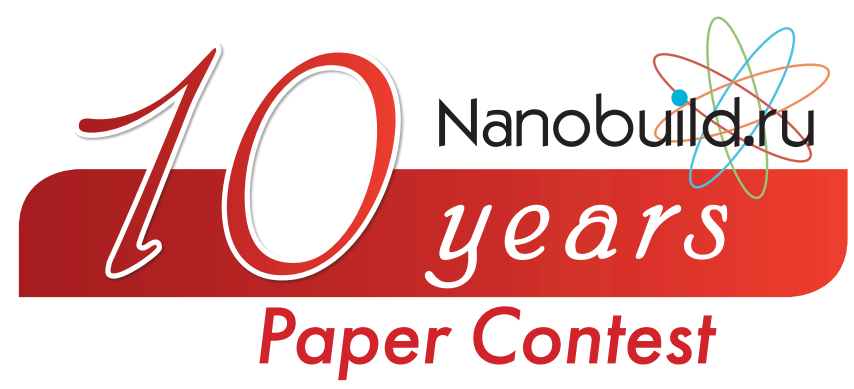

UDC 622.691.48

Author: BAIKOV Igor Ravilevich, Doctor of Engineering, Professor, Full member of Academy of Mining Engineering, Head of Department «Industrial Heat Powering», Ufa State Petroleum Technological University; Kosmonavtov St., 1, Ufa, Bashkortostan Republic, Russia, 450064, pte.ugntu@rusoil.net;

Author: SMORODOVA Olga Viktorovna, PhD in Engineering, Associate Professor of Department «Industrial Heat Powering», Ufa State Petroleum Technological University; Kosmonavtov St., 1, Ufa, Bashkortostan Republic, Russia, 450064, olga_smorodova@mail.ru;

Author: KITAEV Sergey Vladimirovich, Doctor of Engineering, Full Member of International Academy of Engineering, Associate Professor of Department «Transport and Storage of Oil and Gas», Ufa State Petroleum Technological University; Kosmonavtov St., 1, Ufa, Bashkortostan Republic, Russia,450064,svkitaev@mail.ru

\title{
INVESTIGATION OF PROPERTIES OF LIQUID CERAMIC THERMAL INSULATION MATERIALS
}

\section{EXTENDED AbStRaCt:}

Today nanotechnology is widely used in the creation of building materials. In some cases, such technologies allow obtaining materials with improved characteristics. In the domestic and foreign markets the new generation of the building heat insulation materials are represented by liquid ceramic heat-insulating materials manufactured with the help of nanotechnology. Liquid ceramic thermal insulation is a composition of glass evacuated nanospheres based on aqueous solutions of acrylic polymers. It is the technical vacuum inside the nanosphere that explains the high heat-shielding properties of such materials.

To determine reliable thermal conductivity coefficient for thermal insulation paint, the experiment has been planned. The designed plan corresponds to the complete factorial experiment. With 4 influencing factors 16 measurements 
of the density of heat flux through a flat sample of thermal paint with a thickness of $7,5 \mathrm{~mm}$ were made to calculate the coefficient of thermal conductivity. Microprocessor device MTF-MG4 was employed in the measurements.

The article shows the experiment results that determined efficiency of liquid ceramic heat-insulating materials for insulation of exterior walls of buildings and constructions covered by the thermal paint «Isollat-02». The value of the average coefficient of thermal conductivity under the experimental conditions is $0,057 \mathrm{~W} /(\mathrm{m} \times \mathrm{K})$. A linear regression model has been constructed to determine the coefficient under different temperature conditions. The coefficients of the model have been specified.

The calculation of the contribution of thermal insulation paint to the thermal resistance of the enclosing structures was performed. It is demonstrated that the relative efficiency of the thermal insulating paint is higher, the lower the heat-shielding properties of the outer fences of the basic structure. The greatest relative energy effect can be obtained by the thermal insulation of block industrial buildings and metal reservoirs for water, hydrocarbons and other flows of technological and engineering applications.

Key words: thermal insulation, thermal insulation materials, coefficient of thermal conductivity, buildings and structures coating.

MACHINE-READABLE INFORMATION ON CC-LICENSES (HTML-CODE) IN METADATA OF THE PAPER

$<$ a rel="license" href="http://creativecommons.org/licenses/by/4.0/" $><$ img alt="Creative Commons License" style="borderwidth:0" src="https://i.creativecommons.org/l/by/4.0/88x31.png" / $></ \mathrm{a}><$ br $/><$ span xmlns:dct="http://purl.org/dc/ terms/" href="http://purl.org/dc/dcmitype/Text" property="dct:title" rel="dct:type" $>$ Investigation of properties of liquid ceramic thermal insulation materials. $<$ /span $>$ by $<$ a xmlns:cc="http://creativecommons.org/ns\#" href="Nanotehnologii v stroitel'stve $=$ Nanotechnologies in Construction. 2018, Vol. 10, no. 5, pp. 106-121. DOI: dx.doi. org/10.15828/2075-85452018-10-5-106-121." property="cc:attributionName" rel="cc:attributionURL">Baikov I.R., Smorodova O.V., Kitaev S.V. $</$ $\mathrm{a}>$ is licensed under a $<\mathrm{a}$ rel="license" href="http://creativecommons.org/licenses/by/4.0/" $>$ Creative Commons Attribution 4.0 International License $</ \mathrm{a}>$. $<\mathrm{br} />$ Based on a work at $<$ a xmlns:dct="http://purl.org/dc/terms/" href="http://nanobuild. $\mathrm{ru} / \mathrm{en} \_\mathrm{EN} /$ nanobuild-5-2018/" rel="dct:source" $>$ http://nanobuild.ru/en_EN/nanobuild-5-2018/</a $>$. $<$ br $/>$ Permissions beyond the scope of this license may be available at $<$ a xmlns:cc="http://creativecommons.org/ns\#" href="svkitaev@mail.ru" rel="cc:morePermissions" $>$ svkitaev@mail.ru $</ a>$.

\section{References:}

1. Federal'nyy zakon «Ob energosberezhenii i o povyshenii energeticheskoy effektivnosti i o vnesenii izmeneniy v otdel'nyye zakonodatel'nyye akty Rossiyskoy Federatsii» 23.11.2009 N 261-FZ (poslednyaya redaktsiya) [Federal Law «On Energy Saving and on 
Improving Energy Efficiency and on Amending Certain Legislative Acts of the Russian Federation» 23.11.2009 № 261-FZ (last edition)]. (In Russian).

2. Energeticheskaya strategiya Rossii na period do 2030 goda. Utverzhdena rasporyazheniyem pravitel'stva RF №1715-r 13.11.2009 (aktualizirovannaya versiya ot 01.01.2018). [Energy strategy of Russia for the period until 2030. Approved by the decree of the Government of the Russian Federation №1715-r 13.11.2009 (updated version 01.01.2018)]. (In Russian).

3. Emmanuel C. Nsofor, Veera Vijay. Pipe Insulation Model for Energy Conservation in Buildings. Energy Engineering. 2011. Vol. 108. No. 3. Pp. 37-50.

4. Eriksson D., Sunden B. Heat and mass transfer in polyurethane insulated district cooling and heating pipes. Journal of Building physics. 1998. Vol. 22. No. 1. Pp. 110-131.

5. Lotz W.A. Pipe insulation specification process. Heating, Piping, Air Conditioning Engineering. 2003. Vol. 75. No. 10. Pp. 58-59.

6. Baikov I.R., Kitaev S.V., Smorodova O.V., Kolotilov Yu.V. Analysis of the heat-insulating materials properties for pipeline fittings. Polymer Science. Series D. 2018. Vol. 11. no. 1. Pp. 96-98.

7. Akhmyarov T.A., Belyayev V.S., Spiridonov A.V., Shubin I.L. Sistema aktivnogo energosberezheniya s rekuperatsiyey tepla [Active energy saving system with heat recovery]. Energosberezheniye. [Energy saving]. 2013. no 4. Pp. 36-46. In Russian. Available at: https://www.abok.ru/for_spec/articles.php?nid=5551 (Accessed: 03.03.2018).

8. Belyayev V.S. Vysokoeffektivnoye teploizolyatsionnoye i antikorrozionnoye pokrytiye «Izollat» - innovatsionnyy produkt otechestvennogo proizvodstva [Highly effective heatinsulating and anticorrosion coating «Izollat» - an innovative product of domestic production]. Neft'. Gaz. Novatsii. [Oil. Gas. Innovations ]. 2010. no. 5. Pp. 27-31. (In Russian).

9. Loginova N.A. Opredeleniye effektivnosti tonkoplenochnykh teploizolyatsionnykh pokrytiy primenitel'no k sistemam teplosnabzheniya: dis. ... kand. tekhn. Nauk [Determination of the effectiveness of thin-film heat-insulating coatings in relation to heat supply systems: Abstract of PhD thesis.] Moskva, Moskovskiy energeticheskiy institut (TU) [Moscow, Moscow Power Engineering Institute (TU)]. 2010. 133 p. (In Russian).

10. Shevtsov A.N. Zhidkokeramicheskiye teploizolyatsionnyye pokrytiya: drugoy vzglyad [Liquid-ceramic heat-insulating coatings: a different view]. Materialy i tekhnologii. [Materials and technologies]. 2010. no. 4(58). Pp. 20-31. Available at: http://strved.ru/jur/ nomer/2010/04/20.pdf (accessed: 03.03.2018). (In Russian).

11. Kitayev S.V., Smorodova O.V. Matematicheskoye modelirovaniye ispareniya szhizhennykh uglevodorodov pri narushenii teplovoy izolyatsii rezervuara [Mathematical simulation of the evaporation of liquefied hydrocarbons in the event of a thermal insulation of the reservoir]. Neftegazovoye delo: elektron. nauch. jurn. [Oil and gas business: electron. scientific journal]. 2017. no. 1. Pp. 108-120. (In Russian). Available at: http://ogbus.ru/ issues/1_2017/ogbus_1_2017_p108-120_KitaevSV_ru.pdf. (Accessed: 20.07.2017).

12. GOST 7076-99. Materialy i izdeliya v stroitel'stve. Metod opredeleniya teploprovodnosti i termicheskogo soprotivleniya pri statsionarnom teplovom rezhime [State standards 
7076-99. Materials and products in construction. Method for the determination of thermal conductivity and thermal resistance under steady-state thermal conditions. Materials and products in construction. Method for the determination of thermal conductivity and thermal resistance under steady-state thermal conditions]. Moscow, FGUP TSPP. 1999. (In Russian).

13. Obraztsov D.V., Fokin V.M. Issledovaniye prochnostnykh i teplofizicheskikh svoystv nanomodifitsirovannykh stroitel'nykh i teplozashchitnykh materialov [Study of strength and thermal physical properties of nanomodified construction and thermal insulating materials]. Vestnik TGTU [Transactions of TSTU], 2012. V. 18. no. 4. Pp. 1051-1061. Available at: http://vestnik.tstu.ru/rus/t_18/pdf/18_4_041.pdf Accessed 15.09.18. (In Russian).

14. Dekusha L.V., Grishchenko T.G., Vorob'yev L.I. O real'nykh fizicheskikh svoystvakh i vozmozhnostyakh teploizoliruyushchikh krasok [About real physical properties and possibilities of heat-insulating paints]. Promyshlennaya teplotekhnika [Industrial Heat Engineering]. 2006. no. 5. Pp. 93-96. (In Russian).

15. Ivanov I.Ye. Eksperimental'nyye issledovaniya effektivnosti zhidkoy teploizolyatsii po GOST 7076-99[Experimental studies of the effectiveness of liquid heat insulation in accordance with GOST 7076-99] /Nauka i innovatsii v XXI veke: aktual'nyye voprosy, otkrytiya i dostizheniya: sbornik statey IV Mezhdunarodnoy nauchno-prakticheskoy konferentsii. V 3ch. CH.1[Science and innovations in the XXI century: current issues, discoveries and achievements: a collection of articles of the IV International Scientific and Practical Conference. In 3 parts. P. 1]. Penza.: MTSNS «Nauka i prosveshcheniye» [Penza, MCSN «Science and Education»]. 2017. Pp. 41-50. (In Russian).

16. Connor W.S., Young S. Fractional Factorial Designs for Experiments with Factors of two or three Levels. National Bureau of Standards, Applied Mathematics Series, 58, 1961.

17. Fisher R.A. The Design of Experiments. $6^{\text {th }}$ ed., London, Oliver and Boyd, 1951.

18. Vatin N.I., Nemova D.V., Rymkevich P.P., Gorshkov A.S. Vliyaniye urovnya teplovoy zashchity ograzhdayushchikh konstruktsiy na velichinu poter' teplovoy energii v zdaniyakh [Influence of the level of thermal protection of enclosing structures on the amount of thermal energy losses in buildings]. Inzhenerno-stroitel'nyy zhurnal [Engineering and construction journal] - 2012. no. 8 (34). Pp. 4-14. (In Russian).

19. Farkhan M.M., Magaril R.Z. Snizheniye poter' logkikh uglevodorodov pri bol'shikh dykhaniyakh neftyanykh rezervuarov [Reduction of losses of light hydrocarbons at large respiration of oil reservoirs]. Izvestiya vysshikh uchebnykh zavedeniy. Neft' i gaz. [News of higher educational institutions. Oil and Gas]. 2011. no. 5. pp. 83-84. (In Russian).

20. Kireyev I.R., Barakhnina V.B., Latypova G.I. Sovremennyye polimernyye materialy dlya zashchity stal'nykh rezervuarov dlya khraneniya nefti i nefteproduktov ot korrozii [Modern polymeric materials for the protection of steel tanks for storing oil and oil products from corrosion]. Ekologicheskiy vestnik Rossii. [Ecological Herald of Russia] 2017. no. 3. pp. 14-18. (In Russian).

21. Yakushev N.M., Korepanova K.M., Korobeynikova Yu.A. Sravneniye zhidkogo penoizola i zhidkogo keramicheskogo teploizolyatora kak odnogo iz sposobov energoeffektivnogo utepleniya zdaniya [Comparison of liquid foam and liquid ceramic insulator as one of the 
methods of energy-efficient building insulation]. Fotinskiye chteniya. [Fotinsky readings] 2017. no. 1 (7). pp. 158-161. (In Russian).

22. Rozhentsova N.V., Biktimirov Z.M., Galyautdinova A.R. Optimizatsiya svoystv teplozashchity krovli [Optimization of the properties of the thermal protection of the roof]. Aktual'nyye nauchnyye issledovaniya v sovremennom mire. [Actual scientific research in the modern world]. 2018. no. 5-1 (37). pp. 115-118. (In Russian).

23. Fattakhov I.G., Kadyrov R.R., Kuleshova L.S.Metody teploizolyatsii ust'ya nagnetatel'nykh skvazhin [Methods of thermal insulation of the wellheads of injection wells.] // Neftegazovoye delo: elektron. nauch. jurn. [Oil and gas business: electron. scientific journal]. 2012. no. 1. pp. 112-116. [In Russian]. Available at: http://ogbus.ru/files/ogbus/authors/FattakhovIG/FattakhovIG_2.pdf (Accessed: 20.04.2018).

\section{Dear Colleagues!}

THE REFERENCE TO THIS PAPER HAS THE FOLLOWING CITATION FORMAT:

Baikov I.R., Smorodova O.V., Kitaev S.V. Investigation of properties of liquid ceramic thermal insulation materials. Nanotehnologii $\mathrm{v}$ stroitel'stve $=$ Nanotechnologies in Construction. 2018, Vol. 10, no. 5, pp. 106-121. DOI: dx.doi. org/10.15828/2075-8545-2018-10-5-106-121. (In Russian). 


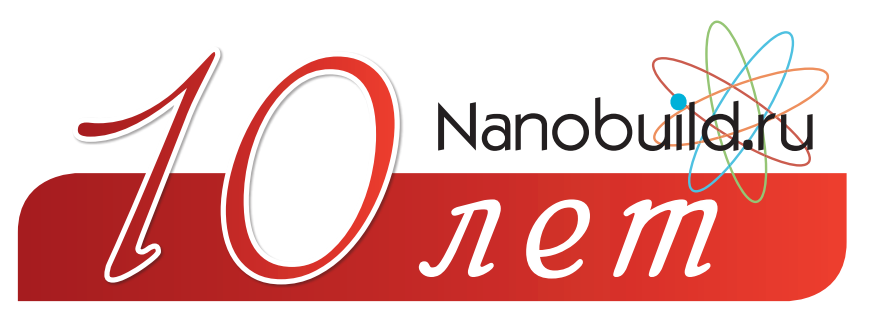

конкурс статей

уДК 622.691.48

Автор: БАЙКОВ Игорь Равильевич, д-р техн. наук, проф., действительный член Академии горных наук, зав. каф. «Промышленная теплоэнергетика», ФГБОУ ВО «Уфимский государственный нефтяной технический университет»; ул.Космонавтов, д. 1, г. Уфа, Республика Башкортостан, 450064, pte.ugntu@rusoil/net;

Автор: СМОРОДоВА Ольга Викторовна, канд. техн. наук, доц. каф. «Промышленная теплоэнергетика», ФГБОУ ВО «Уфимский государственный нефтяной технический университет»; ул. Космонавтов, д. 1, г. Уфа, Республика Башкортостан, 450064, olga_smorodova@mail.ru;

Автор: КИТАЕВ Сергей Владимирович, д-р техн. наук, действительный член Международной инженерной академии, доц. каф. «Транспорт и хранение нефти и газа», ФГБОУ ВО «Уфимский государственный нефтяной технический университет»; ул. Космонавтов, д. 1, г. Уфа, Республика Башкортостан, 450064, svkitaev@mail.ru

\section{ИССЛЕДОВАНИЕ СВОЙСТВ ЖИДКИХ КЕРАМИЧЕСКИХ ТЕПЛОИЗОЛЯЦИОННЫХ МАТЕРИАЛОВ}

\section{АННОТАЦИЯ К СТАТЬЕ (АВТОРСКОЕ РЕЗЮМЕ, РЕФЕРАТ):}

В настоящее время находят широкое применение нанотехнологии при создании строительных материалов. В ряде случаев такие технологии позволяют получить материалы с улучшенными характеристиками. Строительные утепляющие материалы нового поколения на отечественном и зарубежном рынке представлены жидкими керамическими теплоизоляционными материалами, изготавливаемыми с использованием нанотехнологий. Жидкая керамическая теплоизоляция представляет собой композицию стеклянных вакуумированных наносфер на основе водных растворов акриловых полимеров. Именно техническим вакуумом внутри наносфер объясняются высокие теплозащитные свойства таких материалов. 
Для достоверного определения коэффициента теплопроводности теплоизоляционной краски было реализовано планирование эксперимента. Разработанный план соответствует полному факторному эксперименту. При количестве 4 влияющих факторов было проведено 16 измерений плотности теплового потока через плоский образец термокраски толщиной 7,5 мм для расчета коэффициента теплопроводности. Для измерений использован микропроцессорный прибор ИТП-МГ4.

В статье приведены результаты определения эффективности жидких керамических теплоизоляционных материалов для наружных стен зданий и сооружений на примере термокраски марки «Изоллат-02». Экспериментально установлено значение среднего коэффициента теплопроводности в опытных условиях - 0,057 Вт/(м $\times$ К). Построена линейная регрессионная модель для его определения в различных температурных условиях. Определены коэффициенты модели.

Выполнен расчет вклада теплоизоляционной краски в термическое сопротивление ограждающих конструкций. Показано, что относительная эффективность теплоизоляционной краски тем выше, чем ниже теплозащитные свойства наружных ограждений основной конструкции. Наибольший относительный энергетический эффект может быть получен при утеплении краской блочных промышленных зданий и металлических резервуаров воды, углеводородов и прочих потоков технологического и инженерного назначения.

Ключевые слова: термокраска, теплоизоляционные материалы, коэффициент теплопроводности, покрытие зданий и сооружений.

DOI: dx.doi.org/10.15828/2075-8545-2018-10-5-106-121

МАШИНОЧИТАЕМАЯ ИНФОРМАЦИЯ о СС-ЛИЦЕНЗИИ В МЕТАДАННЫХ СТАТЬИ (HTML-КОД):

$<$ a rel="license" href="http://creativecommons.org/licenses/by/4.0/" ><img alt="Creative Commons License" style="borderwidth:0" src="https://i.creativecommons.org/l/by/4.0/88x31.png" / $></ \mathrm{a}><$ br $/><$ span xmlns:dct="http://purl.org/dc/ terms/" href="http://purl.org/dc/dcmitype/Text" property="dct:title" rel="dct:type">Исследование свойств жидких керамических теплоизоляционных материалов $</$ span $>$ by $<$ a xmlns:cc="http://creativecommons.org/ns\#" href="Нанотехнологии в строительстве. - 2018. - Том 10, № 5. - С. 106-121. - DOI: dx.doi.org/10.15828/2075-8545-2018- 10-5-106-121." property="cc:attributionName" rel="cc:attributionURL"> Байков И.Р., Смородова О.В., Китаев С.В. $</$ a $>$ is licensed under a $<$ a rel="license" href="http://creativecommons.org/licenses/by/4.0/" $>$ Creative Commons Attribution 4.0 International License $<$ / a $>$. $<$ br $/>$ Based on a work at $<$ a xmlns:dct="http://purl.org/dc/terms/" href="http://nanobuild.ru/ru_RU/nanobuild-5-2018/" rel="dct:source" $>$ http://nanobuild.ru/ru_RU/nanobuild-5-2018/</a $>$. $<$ br $/>$ Permissions beyond the scope of this license may be available at <a xmlns:cc="http://creativecommons.org/ns\#" href="svkitaev@mail.ru" rel="cc:morePermissions">svkitaev@ mail.ru $</ a>$. 
настоящее время актуальными являются вопросы покрытия и утепления зданий и сооружений для обеспечения требуемого внутреннего теплового режима, которые решаются как при проектировании, так и при эксплуатации объектов. Необходимость реализации энергосберегающих технологий определяется нормативными документами федерального уровня $[1,2]$.

Традиционно для утепления зданий [3], сооружений [4] и трубопроводов [5] применяются такие материалы, как вата минеральная тяжелая, вата хлопковая, пеностекло легкое, засыпная перлитовая теплоизоляция. Коэффициент теплопроводности перечисленных материалов находится в диапазоне от 0,045 до 0,075 Вт/(м×K).

Кроме усиления теплозащитных свойств и оптимизации толщины тепловой изоляции [6] при выборе используемых материалов применяются и активные методы энергосбережения [7] - повышение эффективности теплопотребления зданиями за счет рекуперации тепловой энергии вентвыбросов.

В настоящее время строительные материалы нового поколения на энергетическом рынке представлены жидкими керамическими теплоизоляционными материалами (अ゚КТМ), изготавливаемыми с использованием нанотехнологий [8]. ЗКидкая керамическая теплоизоляция представляет собой композицию стеклянных микрогранул (или микросфер), внутри которых сформирован разреженный газ (технический вакуум) на основе водных растворов акриловых полимеров [9]. Именно вакуумом внутри микросфер объясняются ее высокие теплозащитные свойства [10].

Рассмотрим эффективность применения З๙КТМ на примере термокраски «Изоллат-02». Заявленная заводом-изготовителем долговечность такой термокраски составляет более 15 лет [11], тогда как минеральная вата и засыпная изоляция при эксплуатации сохраняют требуемые теплозащитные свойства в течение периода не более 2-3 лет [12].

Авторами статьи был реализован ряд лабораторных экспериментов [13] по определению основной характеристики теплоизоляционного 
материала - коэффициента теплопроводности - в зависимости от средней температуры материала [14].

Для измерения коэффициента теплопроводности использовался микропроцессорный прибор измеритель плотности теплового потока ИТП-МГ4 [15]. Принцип работы прибора ИТП-МГ4 основан на создании стационарного теплового потока, проходящего через плоский образец определенной толщины и направленного перпендикулярно к лицевым граням образца. Основными контролируемыми при эксперименте параметрами являлись толщина образца, плотность теплового потока и температура противоположных лицевых граней образца.

Стационарная установка для измерения коэффициента теплопроводности состоит из следующих основных элементов:

- блока управления нагревателем;

- $\quad$ холодильника на элементах Пельтье;

- тепломера;

- платиновых датчиков температуры;

- устройства преобразования первичных сигналов датчиков;

- источника питания.

Охлаждение элементов Пельтье осуществляется дутьевым вентилятором. Микропроцессорный электронный блок обеспечивает измерение сигналов датчиков, управление стационарной установкой, индикацию и сохранение результатов измерений.

Вычисление коэффициента теплопроводности по результатам измерений производится вычислительным встроенным устройством прибора по формуле:

$$
\lambda=\frac{\mathrm{H} \cdot \mathrm{q}}{\mathrm{t}_{\mathrm{H}}-\mathrm{t}_{\mathrm{x}}}, \text { В } /\left(\mathrm{M} \cdot{ }^{\circ} \mathrm{C}\right),
$$

где Н - толщина измеряемого образца, м; q - плотность стационарного теплового потока, проходящего через измеряемый образец, Вт/м²; $t_{\text {н }}$ - температура нагретой грани измеряемого образца, ${ }^{\circ} \mathrm{C} ; \mathrm{t}_{\mathrm{x}}$ - температура холодной грани измеряемого образца, ${ }^{\circ} \mathrm{C}$.

Толщина образца и разница температур нагретой и холодной граней выбиралась в зависимости от прогнозируемой теплопроводности материала.

Для проведения измерений коэффициента теплопроводности материала был изготовлен образец из жидко-керамического теплоизоляци- 
онного материала «Изоллат-02» в виде прямоугольного параллелепипеда размером $250 \times 250 \times 7,5$ мм. При толщине измеряемого образца 7,5 мм разность температур холодной и нагретой граней ожидалась в диапазоне $\Delta \mathrm{t}=8 \div 20^{\circ} \mathrm{C}$.

Для оптимизации количества необходимых измерений предварительно выполнено планирование эксперимента по методике [16]. Окончательный план проведения эксперимента приведен в табл. 1.

Таблица 1

\section{План проведения эксперимента}

\begin{tabular}{|c|c|c|c|c|}
\hline \multirow{2}{*}{ Номер опыта } & \multicolumn{4}{|c|}{ Факторы эксперимента } \\
\cline { 2 - 5 } & $\mathbf{t}_{\mathbf{x}},{ }^{\mathbf{}} \mathbf{C}$ & $\mathbf{t}_{\mathbf{H}},{ }^{\circ} \mathbf{C}$ & $\Delta \mathbf{t},{ }^{\circ} \mathbf{C}$ & $\mathbf{q}, \mathbf{B} \mathbf{T}$ \\
\hline 1 & 5 & 25 & 20 & $\mathrm{q}_{\max }$ \\
\hline 2 & 17 & 25 & 8 & $\mathrm{q}_{\min }$ \\
\hline 3 & 25 & 45 & 20 & $\mathrm{q}_{\max }$ \\
\hline
\end{tabular}

Составленный план соответствует полному факторному эксперименту [17]. При его реализации необходимо выполнить несколько измерений при условиях:

- $\quad$ количество измерений составляет $2^{\mathrm{n}}$, где $\mathrm{n}$ - количество факторов;

- каждый фактор принимает только два значения - верхнее и нижнее;

- в процессе измерения верхние и нижние значения факторов комбинируются во всех возможных сочетаниях.

Преимуществами полного факторного эксперимента являются следующие достоинства:

- $\quad$ простота решения системы уравнений для оценки параметров;

- статистическая избыточность количества измерений, которая

уменьшает влияние погрешностей отдельных измерений на оценку параметров.

При проведении эксперимента по измерению коэффициента теплопроводности ЗћКТМ «Изоллат» для набора достаточного количества опытных данных в рамках обозначенной области было проведено $2^{\mathrm{n}}=2^{4}=16$ измерений при граничных значениях всех влияющих факторов во всех возможных сочетаниях. 
На рис. 1 приведена полученная зависимость коэффициента теплопроводности исследуемого материала от средней его температуры (маркерами показаны экспериментальные точки).

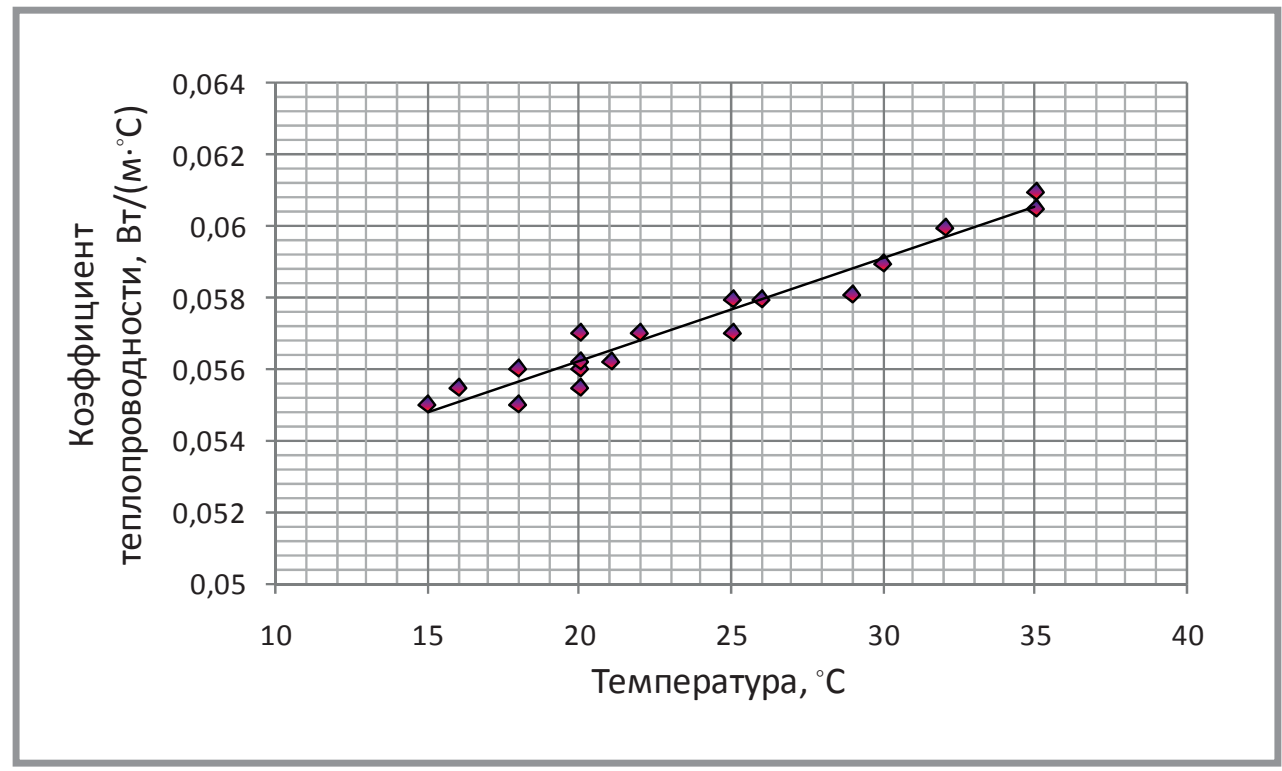

Рuc. 1. Зависимость коэффициента теплопроводности «Изоллат-02» от температуры

Как следует из рис. 1 , зависимость теплопроводности исследуемого материала от его температуры линейна, поэтому для ее аналитического представления примем уравнение вида:

$\lambda=\lambda_{0}+\mathrm{b} \cdot \mathrm{t}$,

где $\lambda_{0}-$ эмпирические коэффициенты; $\mathrm{t}-$ температура, ${ }^{\circ} \mathrm{C}$.

В условиях проводимого эксперимента за температуру материала (t) в каждом опыте принималась средняя температура горячей и холодной грани исследуемого образца.

По результатам применения метода наименьших квадратов для определения коэффициентов регрессии уравнение (2) для термокраски «Изоллат-02» приняло вид:

$$
\lambda=0,0505+0,0003 \cdot \mathrm{t} .
$$

Степень адекватности аппроксимации экспериментальных данных составила $\mathrm{R}^{2}=0,95$. 
Среднее значение коэффициента теплопроводности по результатам измерений составило $0,057 \mathrm{BT} /\left(\mathrm{m}^{\circ}{ }^{\circ} \mathrm{C}\right)$, что с отклонением не более $18 \%$ соответствует значению показателя оригинальной краски марки Mascoat (производство США) - 0,0698 Вт $/\left(\mathrm{м}^{\circ}{ }^{\circ} \mathrm{C}\right)$. Таким образом, экспериментально было установлено, что по теплозащитным свойствам термокраска «Изоллат-02» не уступает традиционным теплоизоляционным материалам.

Для оценки эффективности использования жЖКТМ в покрытиях зданий и сооружений было выполнено моделирование процессов теплообмена через наружные конструкции [18] в 4-х вариантах - три варианта покрытия наружных стен промышленных и жилых зданий и один вариант покрытия металлических технологических резервуаров (табл. 2).

Таблица 2

\section{Исходные данные для моделирования теплопередачи через наружные ограждения зданий и сооружений с использованием термокраски «Изоллат»}

\begin{tabular}{|c|c|c|c|c|}
\hline \multirow{3}{*}{$\begin{array}{c}\text { Вариант применения } \\
\text { жидкокерамической } \\
\text { краски }\end{array}$} & \multicolumn{2}{|c|}{ Базовый слой } & \multicolumn{2}{|c|}{ Слой термокраски } \\
\hline & $\begin{array}{c}\text { толщина } \\
\text { слоя }\end{array}$ & $\begin{array}{c}\text { коэффициент } \\
\text { теплопрово- } \\
\text { дности }\end{array}$ & $\begin{array}{c}\text { толщина } \\
\text { слоя }\end{array}$ & $\begin{array}{c}\text { коэффициент } \\
\text { теплопрово- } \\
\text { дности }\end{array}$ \\
\hline & MM & Вт $/(\mathbf{M} \times \mathbf{K})$ & MM & $\mathbf{B T} /(\mathbf{M} \times \mathbf{K})$ \\
\hline $\begin{array}{l}\text { Вариант 1. Произ- } \\
\text { водственное здание } \\
\text { панельной } \\
\text { конструкции }\end{array}$ & 380 & 0,70 & 7,5 & 0,055 \\
\hline $\begin{array}{l}\text { Вариант } 2 . \text { Ж⿱илое } \\
\text { здание из силикат- } \\
\text { ного кирпича }\end{array}$ & 650 & 0,70 & 4,0 & 0,055 \\
\hline $\begin{array}{l}\text { Вариант 3. Жиллее } \\
\text { здание из керамиче- } \\
\text { ского кирпича }\end{array}$ & 650 & 0,57 & 4,0 & 0,055 \\
\hline $\begin{array}{l}\text { Вариант } 4 . \text { Металли- } \\
\text { ческий резервуар }\end{array}$ & 30 & 60,0 & 2,0 & 0,055 \\
\hline
\end{tabular}


Результаты расчетов показали (рис. 2), что наибольшего эффекта при использовании ЖККТМ «Изоллат» в строительстве можно достигнуть при наружном утеплении промышленных зданий из строительных панелей (вариант 1 в табл. 2 и на рис. 2) - теплопотребление здания будет снижено на $25 \%$.

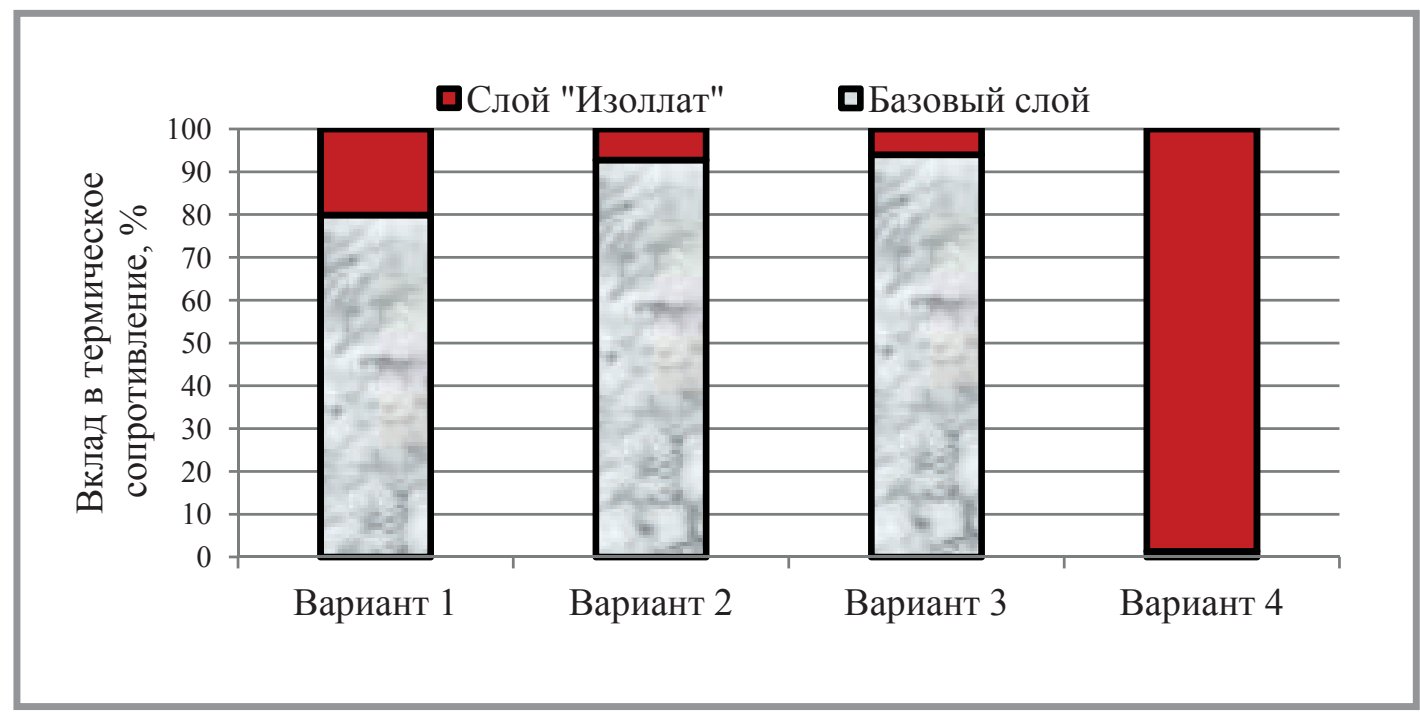

Рис. 2. Эффект от применения жидкокерамического материала «Изоллат»

Максимальный эффект от использования «Изоллат» можно получить при его нанесении на металлические поверхности, обладающие минимальным собственным термическим сопротивлением. Например, при нанесении ЖККТМ на резервуары хранения нефти и нефтепродуктов на производственных площадках системы добычи и магистрального транспорта нефти будут существенно снижены потери хранимого продукта от испарений при больших и малых дыханиях резервуаров [19].

Кроме высоких теплозащитных характеристик, термокраскам присущи дополнительные полезные в эксплуатации свойства. В зависимости от сферы применения разработано несколько марок покрытия «Изоллат». В состав каждой из них включены специальные наноструктурные компоненты, обеспечивающие высокое качество, сейсмоустойчивость, антикоррозионную защиту [20] и хорошую адгезию к любым материалам.

К положительным свойствам термокрасок относятся также возможность нанесения на поверхности сложной геометрической формы, 
звукоизоляционные и противопожарные свойства, благодаря которым они могут широко применяться при утеплении стен [21] и кровли зданий [22], трубопроводной арматуры [23] и другого промышленного оборудования.

\section{Выводы:}

1. С целью определения теплопроводности термокраски на основе обработки экспериментальных данных (16 измерений прибором ИТП-МГ4) установлено среднее значение коэффициента теплопроводности материала «Изоллат-02» в условиях эксперимента $0,057 \mathrm{BT} /(\mathrm{M} \times \mathrm{K})$.

2. Установлен линейный вид функциональной зависимости коэффициента теплопроводности термокраски «Изоллат-02» от температуры.

3. Выполнена оценка вклада термокраски «Изоллат» в суммарное термическое сопротивление ограждающей конструкции. Показано, что наибольший относительный энергетический эффект может быть получен при утеплении панельных промышленных зданий (до $25 \%$ ) и покрытии металлических резервуаров с дополнительным противокоррозионным эффектом.

\section{Библиографический список:}

1. Федеральный закон «Об энергосбережении и о повышении энергетической эффективности и о внесении изменений в отдельные законодательные акты Российской Федерации» от 23.11.2009 № 261-ФЗ (последняя редакция).

2. Энергетическая стратегия России на период до 2030 года. Утверждена распоряжением правительства РФ № 1715-р от 13.11.2009 (актуализированная версия от 01.01.2018).

3. Emmanuel $C$. Nsofor \& Veera Vijay Pipe Insulation Model for Energy Conservation in Buildings // Energy Engineering. 2011. Vol. 108. No. 3. Pp. 37-50.

4. Eriksson D., Sunden B. Heat and mass transfer in polyurethane insulated district cooling and heating pipes // Journal of Building physics. 1998. Vol. 22. No. 1. Pp. 110-131.

5. Lotz W.A. Pipe insulation specification process // Heating, Piping, Air Conditioning Engineering. 2003. Vol. 75. No. 10. Pp. 58-59. 
6. Baikov I.R., Kitaev S.V., Smorodova O.V., Kolotilov Yu.V. Analysis of the heat-insulating materials properties for pipeline fittings//Polymer Science. Series D. 2018. T. 11. № 1. C. 96-98.

7. Ахмяров Т.А., Беляев В.С., Спиридонов А.В., Шубин И.Л. Система активного энергосбережения с рекуперацией тепла // Энергосбережение. - 2013. - № 4. - C. 36-46. - URL: https://www.abok.ru/for_spec/articles.php?nid=5551 (дата обращения: 03.03.2018).

8. Беляев В.С. Высокоэффективное теплоизоляционное и антикоррозионное покрытие «Изоллат» - инновационный продукт отечественного производства // Нефть. Газ. Новации. - 2010. - № 5. - С. 27-31.

9. Логинова Н.А. Определение эффективности тонкопленочных теплоизоляционных покрытий применительно к системам теплоснабжения: дис. ... канд. техн. наук. - Москва: Московский энергетический институт (ТУ), 2010. - 133 с.

10. Шевцов А.Н. Жииккокерамические теплоизоляционные покрытия: другой взгляд // Материалы и технологии. - 2010. - № 4 (58). - C. 20-31. - URL: http://strved.ru/jur/ nomer/2010/04/20.pdf (дата обращения: 03.03.2018).

11. Китаев С.В., Слородова О.В. Математическое моделирование испарения сжиженных углеводородов при нарушении тепловой изоляции резервуара / Нефтегазовое дело. 2017. - № 1. - C. 108-120. - URL: http://ogbus.ru/issues/1_2017/ogbus_1_2017_ p108-120_KitaevSV_ru.pdf. (дата обращения: 20.07.2017).

12. ГОСТ 7076-99. Материалы и изделия в строительстве. Метод определения теплопроводности и термического сопротивления при стационарном тепловом режиме - М.: ФГУП ЦПП, 1999.

13. Образиов Д.В., Фокин В.М. Исследование прочностных и теплофизических свойств наномодифицированных строительных и теплозащитных материалов // Вестник ТГТУ, 2012. - T. 18, № 4. - С. 1051-1061.

14. Декуша Л.В., Грищенко Т.Г., Воробьев Л.И. О реальных физических свойствах и возможностях теплоизолирующих красок // Промышленная теплотехника. - 2006. № 5. - С. 93-96.

15. Иванов И.Е. Экспериментальные исследования әффективности жидкой теплоизоляции по ГОСТ 7076-99 // Наука и инновации в XXI веке: актуальные вопросы, открытия и достижения: сборник статей IV Международной научно-практической конференции. В 3 ч. - Пенза: МЦНС «Наука и просвещение», 2017. - Ч. 1. - С. 41-50.

16. Connor W.S., Young S. Fractional Factorial Designs for Experiments with Factors of two or three Levels. National Bureau of Standards, Applied Mathematics Series, 58, 1961.

17. Fisher R.A. The Design of Experiments. $6^{\text {th }}$ ed., London, Oliver and Boyd, 1951.

18. Ватин Н.И., Нелова Д.В., Рылккевич П.П., Горшков А.С. Влияние уровня тепловой защиты ограждающих конструкций на величину потерь тепловой энергии в зданиях // Инженерно-строительный журнал. - 2012. - № 8 (34). - С. 4-14.

19. Фархан M.M., Магарил Р.З. Снижение потерь лёгких углеводородов при больших дыханиях нефтяных резервуаров // Известия высших учебных заведений. Нефть и газ. 2011. - № 5. - C. 83-84. 
20. Киреев И.Р., Барахнина В.Б., Латыпова Г.И. Современные полимерные материалы для защиты стальных резервуаров для хранения нефти и нефтепродуктов от коррозии // Экологический вестник России. - 2017. - № 3. - С. 14-18.

21. Якушев Н.М., Корепанова К.М., Коробейникова Ю.А. Сравнение жидкого пеноизола и жидкого керамического теплоизолятора как одного из способов энергоэффективного утепления здания // Фотинские чтения. - 2017. - № 1 (7). - С. 158-161.

22. Роженцова Н.В., Биктилиров З.М., Галяутдинова А.Р. Оптимизация свойств теплозащиты кровли // Актуальные научные исследования в современном мире. - 2018. № 5-1 (37). - С. 115-118.

23. Фаттахов И.Г., Кадыров Р.Р., Кулешова Л.С. Методы теплоизоляции устья нагнетательных скважин // Нефтегазовое дело. - 2012. - № 1. - C. 112-116. - URL: http://ogbus. ru/files/ogbus/authors/FattakhovIG/FattakhovIG_2.pdf (дата обращения: 20.04.2018).

\section{УВАЖАЕМЫЕ КОЛЛЕГИ!}

ПРИ ИСПОЛЬЗОВАНИИ МАТЕРИАЛА ДАННОЙ СТАТЬИ ПРОСИМ ДЕЛАТЬ БИБЛИОГРАФИЧЕСКУЮ ССЫЛКУ НА НЕЁ:

Байков И.Р., Слородова О.В., Китаев С.В. Исследование свойств жидких керамических теплоизоляционных материалов // Нанотехнологии в строительстве. 2018. - Tом 10, № 5. - C. 106-121. - DOI: dx.doi.org/10.15828/2075-8545-201810-5-106-121.

\section{Dear Colleagues!}

THE REFERENCE TO THIS PAPER hAS THE FOLLOWING CITATION FORMAT:

Baikov I.R., Smorodova O.V., Kitaev S.V. Investigation of properties of liquid ceramic thermal insulation materials. Nanotehnologii $\mathrm{v}$ stroitel'stve = Nanotechnologies in Construction. 2018, Vol. 10, no. 5, pp. 106-121. DOI: dx.doi. org/10.15828/2075-8545-2018-10-5-106-121. (In Russian). 